\title{
Beiträge zur Kenntnis der Antipeptone.
}

\author{
Von \\ Fritz Mulller.
}

(Aus der chemischen Abteilung des physiologischen Instituts der Universität Leipzig. (Der Redaktion zugegangen am 18. April 1803.)

M. Siegfried hat unter den tryptischen Verdauungsprodukten des Eiweißes mit Hilfe seiner Eisenmethode zwei Antipeptone isoliert, 1 ) die er als Antipepton $\alpha$ und Antipepton $\beta$ bezeichnet und denen die einfachsten, dem Äquivalentgewichte entsprechenden Formeln $\mathrm{C}_{10} \mathrm{H}_{17} \mathrm{~N}_{3} \mathrm{O}_{5}$ bez. $\mathrm{C}_{11} \mathrm{~N}_{19} \mathrm{~N}_{3} \mathrm{O}_{5}$ zukommen.

Die Antipeptone haben sich als optisch aktiv erwiesen, und zwar drehen sie die Ebene des polarisierten Lichts nach links.

Als Spaltungsprodukte, die Siegfried bei der Zersetzung mit siedender Säure erhielt, sind Lysin, Glutaminsäure und Asparaginsäure zu erwähnen. Die Enstehung von Arginin war zweifelhaft.

Als ich, einer freundlichen Aufforderung des Herrn Prof. Sieg fried Folge leistend, mich dem Studium der Antipeptone zu widmen begann, fiel mir die Aufgabe zu, zunächst die Methode nochmals auf ihre absolute Sicherheit in der Anwendung auch auf andere Eiweißkörper als Witte-Pepton, das in den vorwiegenden Fällen als Ausgangsmaterial zu den Darstellungen der Antipeptone benutzt worden war, zu prüfen. Dann sollte versucht werden, Konstanz der Werte für das spez. Drehvermögen der Antipeptone zu erlangen. Das spezifische Drehvermögen

1) M. Siegfried, Diese Zeitschrift, Bd. XXXV S. 164. - Berichte der deutsch. chem. Ges. Bd. 33 S. 2851. 
der Peptone schien nämlich «ein scharfes Kriterium für die Reinheit der Peptone und für die Charakteristik derselben und ihrer Muttersubstanzen, der verschiedenen Eiweißkörper» abzugeben.

Wichtig war die Beantwortung der Frage, ob bei der Spaltung der Antipeptone Arginin entsteht. Einmal scheint nach den Untersuchungen von Kossel und Kutscher, die bis jetzt bei jeder Eiweißspaltung Arginin gefunden haben, das Arginin ein für die Eiweißnatur eines Körpers charakteristisches Spaltungsprodukt zu sein und zweitens gibt das Auffinden von Arginin unter den Spaltungsprodukten ohne weiteres darüber Aufschluß, daß dem Antipepton die einfache Molekulargröße nicht zugeschrieben werden kann; das Argininmolekül enthält bekanntlich 4 Stickstoffatome, die einfache Formel der Antipeptone dagegen bloß drei.

\section{A. Verdauung von W. Kühnes Antialbumid.}

Zunächst sollte versucht werden, Antipepten aus Antialbumid darzustellen. Antialbumid nannte bekanntlich W. Kühne den gegen verdünnte, siedende Säure widerstandsfähigen Eiweißkörper, welcher nach seinen Untersuchungen der Antigruppe zugehört und der bei der tryptischen Verdauung ausschließlich in Antipepton, ohne gleichzeitige Entstehung niederer Spaltungsprodukte, übergehen soll.

W. Kühne hat s. Zt. mündlich Herrn Prof. Siegfried gegenüber die Bitte ausgesprochen, er möchte seine Untersuchungen, ob das Antialbumid bei der tryptischen Verdauung glatt reines Antipepton liefert, gelegentlich weiter verfolgen.

Zunächst sei kurz auf Kühnes und Chittendens Darstellung von Antialbumid eingegangen. - Die beiden Forscher unterwarfen teils Hühnereiweiß und teils Fibrin der Einwirkung von 3-5\% iger Schwefelsäure bei $100^{\circ}$ und erhielten eine unlösliche, gallertartige Masse, die von Pepsin in salzsaurer Lösung nicht verdaut warde, in Soda von $0,5 \%$ aber leicht löslich war. 
Als charakteristisches Merkmal ergab sich, daß ein Zusatz von Trypsin zu dieser Lösung dieselbe binnen 2 Stunden zur Gallerte erstarren ließ. Mit fortschreitender Verdauung löste sich die Gallerte wieder bis auf einen Antialbumidrest. Aus dieser Lösung wurde nun mit Alkohol ein strohgelbes Pulver gefällt, das Kühne Antipepton nannte. Daß es natürlich kein reines Antipepton im jetztigen Sinne sein konnte, dürfte ohne weiteres klar sein. Zudem erhielt das Produkt selbst nach 14 tägiger Reinigung durch Dialyse noch 20-30\% Asche.

Es sollte nun untersucht werden, ob sich aus dem so gewonnenen Kühneschen Antipepton mit Hilfe der Siegfriedschen Eisenmethode reines Antipepton darstellen lasse.

$\mathrm{Zu}$ dem Zwecke begann ich nach Kühnes Vorschrift ${ }^{1}$ ) Antialbumid aus Hühnereiweiß darzustellen. Die Einzelheiten des Verfahrens seien in den Hauptzügen wiederholt:

Das Weiße von 50 frischen Eiern wurde zu Schaum geschlagen, nach 24stündigem Stehen von Membranen befreit und koaguliert.

Das Koagulum wurde nach dem Waschen und Abpressen mit $1500 \mathrm{ccm}$ Wasser und $7 \mathrm{ccm}$ engl. Schwefelsäure im Kolben verteilt und 10 Stunden lang im Wasserbad unter möglichstem Luftabschluß auf $100^{\circ}$ erhitzt. Das wenig veränderte Eiweiß wurde von der Säure getrennt und nun mit $310,5 \%$ iger Schwefelsäure 20 Stunden lang auf $100^{\circ}$ erhitzt.

Das entstandene Antialbumid, eine graue Gallerte, wurde gewaschen, zur weiteren Reinigung mit $500 \mathrm{ccm} 0,4 \%$ iger Salzsäure versetzt und 3-5 Tage im Verdauungsbad der Einwirkung von $5 \mathrm{~g}$ Pepsin-Grübler unterworfen. Die Gallerte blieb ungelöst; sie wurde durch Kolieren von der Säure befreit und gewaschen. Das Waschen der gallertartigen Niederschläge ging äußerst langsam von statten, wurde aber sorgfältig betrieben. Aus 50 Eiweißen wurden so in vier analogen Darstellungen je 25-30 g trockenes Antialbumid gewonnen.

Das Antialbumid verhielt sich ganz wie das Kühnesche Präparat. Die Gallerte wurde in $110,5 \%$ iger Soda gelöst

1) Kühne und Ghittenden, Zeitschr. für Biologie Bd. 19 S. 165. 
und mit $5 \mathrm{~g}$ Trypsin-Grübler bei $40^{\circ}$ verdaut. Es trat stets das charakteristische Antialbumidgerinnsel auf, das sich im Verlauf der Verdauungszeit, die zwischen 5 und 7 Tagen schwankte, bis auf unbedeutende Reste wieder löste. - Diese Lösung wurde zur Entfernung nur gelösten Antialbumids mit Essigsäure neutralisiert und erwärmt, wobei sich geringe Mengen Antialbumid wieder abschieden, filtriert und im Vacuum zum Sirup eingedampft. Die Fällung mit Alkohol ergab ein strohgelbes Pulver; im Filtrat wurde durch Zusatz von Äther ein weiterer Niederschlag erhalten. Die Gesamtausbeute aus vier Darstellungen betrug $30 \mathrm{~g}$. Das nicht dialysierte Präparat enthielt 42,7\% Asche.

Dieses «Antipepton» gab deutliche Biuretreaktion; die Millonsche Reaktion war negativ.

Zur Weiterbehandlung wurden die $30 \mathrm{~g}$ Substanz bei $40^{\circ}$ mit Ammonsulfat gesättigt und in neutraler, saurer und ammoniakalischer Lösung je 24 Stunden stehen gelassen; dabei schied sich eine beträchtliche Menge Albumose ab. Diese wurde abfiltriert und im Filtrat nun nach M. Siegfrieds Methode mit Eisenammoniakalaun versetzt. Die aus stark saurer, bez. mit Ammoniak abgestumpfter Lösung erhaltenen Eisenniederschläge I und II wurden nach Vorschrift gewaschen, mit Baryt zersetzt, der Überschuß mit Ammoncarbonat entfernt und im Vacuum bei $40^{\circ}$ eingedampft. Die so erhaltenen Sirupe wurden mit Essigsäure versetzt und so viel Alkohol zugegeben, daß der entstehende Niederschlag eben wieder verschwand. Bein Verrühren dieser Lösungen wurden weiße, flockige Niederschläge erhalten, deren Menge jedoch äußerst gering war, was bei der nachgewiesenen starken Verunreinigung des Kühneschen Antipeptons mit Albumose und dem hohen Aschegehalt nicht überraschen konnte.

Das von Kühne aus Antialbumid gewonnene «Antipepton * ist also kein geeignetes Material zur Darstellung von reinem Antipepton. - Ich gab somit diesen Weg der Antipeptongewinnung auf und begann mit der tryptischen Verdauung von Fibrin. 


\section{B. Darstellung der Antipeptone ans Fibrin.}

Die Methode Siegfrieds verlangt peinliche Befolgung der Vorschriften, wenn man sicher sein will, reines Pepton zu gewinnen.

Nach einigen kleineren Vorversuchen wurden $10 \mathrm{~kg}$ feuchtes, gut gewaschenes Fibrin mit 15 l 0,3\% iger Sodalösung und $7 \mathrm{~g}$ Trypsin-Grübler in 2 Kolben zur Verdauung angesetzt. Durch Zusatz von Chloroform und Thymol wurde gut vor Fäulnis geschützt. Nach Verlauf von 8 Tagen wurden weitere $15 \mathrm{~g}$ Trypsin zugesetzt und nun das Ganze unter steter Kontrolle 4 Wochen verdauen gelassen. Die Sättigung einer Probe mit Ammonsulfat ergab alsdann nur noch eine geringe Abscheidung von Albumose und die Verdauung konnte somit abgebrochen werden. Die Verdauungslösung wurde ganz nach Vorschrift mit Schwefelsäure neutralisiert, nach 24 Stunden filtriert und durch Sättigen mit Ammonsulfat in saurer, neutraler und ammoniakalischer Lösung von der abgeschiedenen Albumose befreit. In der sauren, ammonsulfatgesättigten Lösung wurde die Eisenfällung I mit ammonsulfatgesättigter Eisenammoniakalaunlösung ausgeführt und vom Niederschlag filtriert. Im Filtrat wurde die «Zwischenfällung durch weiteren $\mathrm{Zu}$ satz von Eisenammoniakalaunlösung und Neutralisation mit konzentriertem wässerigen Ammoniak gemacht. Nach deren Entfernung wurde so lange unter stetem Rühren feingepulverter Eisenammoniakalaun eingetragen und die entstehende saure Lösung mit konzentriertem Ammoniak unter Vermeidung neutraler Reaktion abgestumpft, bis eine Probe des Filtrats nur noch schwache Biuretreaktion gab.

Beide Eisenniederschläge wurden nun nach Vorschrift weiter verarbeitet. Sie wurden mehrfach mit gesättigter Ammonsulfatlösung verrieben und abgenutscht. Dann wurde Eisenniederschlag I durch Verrühren mit Wasser, Lösen in Ammoniak und Sättigen des Filtrats vom entstandenen Eisenoxydhydrat mit Ammonsulfat in neutraler, saurer und ammoniakalischer Lösung von den letzten Resten der Albumose befreit, wieder frisch gefällt und gut gewaschen. 
Der Eisenniederschlag II wurde in gesättigter Ammonsulfatlösung verrührt, durch Zusatz von konzentrierter Schwefelsäure in Lösung gebracht und durch Abstumpfen mit konzentriertem Ammoniak wieder ausgefällt und gewaschen. - Beide Eisenniederschläge wurden nun mit Baryt unter Vermeidung eines größeren Überschusses zersetzt und das Filtrat mit Ammoncarbonat vom Baryt befreit. Die so erhaltenen Peptonlösungen wurden im Vacuum bei $40^{\circ}$ eingedampft, der entstehende Sirup mit 12\% iger Essigsäure versetzt und soviel Alkohol zugegeben, daß sich der entstehende Niederschlag eben wieder löste. Die filtrierte Lösung wurde in Alkohol verrührt. Während nun bei dieser Darstellung $A$ aus der Peptonlösung $\beta$ ein weißer, flockiger Niederschlag fiel, der bei geringem Aschegehalt analysenrein war, gelang es mir bei diesem Versuche nicht, aus der Peptonlösung $\alpha$ ein reines Präparat zu erhalten. Die Ausbeute war zu gering, um dasselbe reinigen zu können.

Inzwischen waren weitere $15 \mathrm{~kg}$ feuchtes Fibrin verdaut und analog behandelt worden. Diese Darstellung B ergab sowohl reine, als auch aschefreie Antipeptone in genügender Ausbeute. An völlig reinem Antipepton $\beta$ wurden $18,5 \mathrm{~g}$, an Antipepton a $60 \mathrm{~g}$ gewonnen.

Schließlich wurde bei einer neuen Darstellung die Methode modifiziert.

Zum Versuch wurden $100 \mathrm{~g}$ Wittepepton in 31 0,3\% iger Soda gelöst und mit $3 \mathrm{~g}$ Trypsin verdaut. Von der Überlegung ausgehend, daß das mühsame und zeitraubende Auswaschen der Eisenniederschläge beim Arbeiten in alkoholischer, ammonsulfatgesättigter Lösung schneller vor sich gehen würde, wurde ein Teil der Verdauungslösung mit dem gleichen Volumen Äthylalkohol versetzt und darauf mit Ammonsulfat gesättigt. Am anderen Tage hatte sich eine ölige Schicht von alkoholischem Ammonsulfat abgeschieden, die auch bei der Verminderung des Alkoholgehalts bis auf $30 \%$ bestehen blieb. Da 
beide Schichten peptonhaltig waren, war somit Äthylalkohol nicht $\mathrm{zu}$ verwenden.

Deshalb wurde zu dem Rest der Verdauungslösung' ein gleiches Volumen gesättigter Ammonsulfatlösung und ein Volumen Methylalkohol gegeben, und die Fällung der Eisenniederschläge in dieser Lösung ausgeführt. In der Tat fielen die Niederschläge so, daß sie sich bedeutend leichter - mit einer Mischung von 2 Teilen halbgesättigter Ammonsulfatlösung und 1 Teil Methylalkohol - auswaschen ließen, sodaß für Arbeiten mit größeren Portionen eine beträchtliche Zeitersparnis zu erwarten ist.

Die Zersetzung des Eisenniederschlags II dieser Portion wurde zu Ende geführt und lieferte 2,5 g Antipepton $\alpha$. Das Präparat war nach einmaligem Umfällen analysenrein. Es gab den dem Antipepton $\alpha$ entsprechenden Wert für das spez. Drehvermögen (S. 277).

\section{Zusammensetzung und Eigenschaften der Antipeptone.}

Die Antipeptone wurden sämtlich bei $70^{\circ}$ im Luftbad getrocknet. In der Regel wurde nach 10-14 Tagen Konstanz erreicht.

a) Antipeptone $\beta$ aus Eisenniederschlag I.

Darstellung A:

1. 0,2002 g S. g. $\left\{\begin{array}{l}0,3483 \text { g CO}_{2}=48,03 \% \mathrm{C} \\ 0,1248 \mathrm{~g} \mathrm{H}_{2} \mathrm{O}=7,07 \% \mathrm{H}\end{array}\right.$

2. 0,1862 g S. g. $25 \mathrm{ccm} \mathrm{N}$ bei $100^{\circ}$ und $738 \mathrm{~mm}$ Bar.

$\mathrm{N}=15,85 \%$. - Aschegehalt 1,1\% .

Darstellung B: das Präparat war völlig aschefrei.

3. 0,2248 g S. g. $\left\{\begin{array}{l}0,3996 \mathrm{~g} \mathrm{CO}_{2}=48,48 \% \mathrm{C} \\ 0,1436 \mathrm{~g} \mathrm{H}_{2} \mathrm{O}=7,13 \% \mathrm{H}\end{array}\right.$

4. 0,1738 g S. g. $\left\{\begin{array}{l}0,3076 \mathrm{~g} \mathrm{CO}_{2}=48,27 \% \mathrm{C} \\ 0,1103 \mathrm{~g} \mathrm{H}_{2} \mathrm{O}=7,08 \% \mathrm{H}\end{array}\right.$

5. $0,2205 \mathrm{~g} \mathrm{~S}$. g. $28,5 \mathrm{ccm} \mathrm{N}$ bei $20^{\circ}$ und $761 \mathrm{~mm}$ Bar. $\mathrm{N}=15,08 \%$.

Dieses Präparat wurde dreimal durch Lösen in Wasser und Eintragen in Alkohol umgefällt. - Zur Umfällung gelangten $8 \mathrm{~g}$ Antipepton $\beta$. 
Die Ausbeute nach der 1. Umfällung betrug $6,6 \mathrm{~g}=82 \%$.

$5 \mathrm{~g}$ hiervon wurden zur 2. Umfällung verwendet. Die Ausbeute war $3,0 \mathrm{~g}=60 \%$.

$2 \mathrm{~g}$ der 2. Umfällung wurden zur 3 . Umfällung benutzt. Die Ausbeute betrug $1,3 \mathrm{~g}=65 \%$.

In den Mutterlaugen blieben somit:

bei der 1 . Umfällung $1,4 \mathrm{~g}=18 \%$

bei der 2. Umfällung $2 \mathrm{~g}=40 \%$

bei der 3. Umfällung $0,7 \mathrm{~g}=35 \%$

Die 3. Umfällung wurde analysiert.

6. 0,2335 g S. g. $\left\{\begin{array}{l}0,4122 \mathrm{~g} \mathrm{CO}_{2}=48,15 \% \mathrm{C} \\ 0,1500 \mathrm{~g} \mathrm{H}_{2} \mathrm{O}=7,20 \% \mathrm{H}\end{array}\right.$

7. 0,1470 g S. g. $19,3 \mathrm{ccm} \mathrm{N}$ bei $19,5^{\circ}$ und $759 \mathrm{~mm}$ Bar.

$\mathrm{N}=15,31 \%$.

Die Zusammensetzung des Antipeptons $\beta$ war also auch nach dreimaligem Umfällen dieselbe geblieben. Hieraus ist von neuem bestätigt, daß das zur Umfällung verwendete Antipepton ein einheitlicher Körper ist; wäre es ein Gemisch verschiedener Körper, so müßte bei dem hohen Prozentsatz der in der Mutterlauge verbleibenden Anteile notwendig eine Änderung der Zusammensetzung eintreten.

\begin{tabular}{|c|c|c|c|c|c|c|c|}
\hline & \multicolumn{6}{|c|}{ Gefundene Prozente } & \multirow{3}{*}{\begin{tabular}{|c|} 
Berechnete \\
$\begin{array}{c}\text { Prozente } \\
\text { für }\end{array}$ \\
$\mathrm{C}_{\mathbf{1 1}} \mathrm{N}_{3} \mathrm{H}_{19} \mathrm{O}_{5}$ \\
\end{tabular}} \\
\hline & \multicolumn{2}{|c|}{ Präparat A. } & \multicolumn{3}{|c|}{ Präparat B. } & $\begin{array}{l}\text { Präparat B. } \\
\text { III. Umfäll. }\end{array}$ & \\
\hline No. & 1. & 2. & 3. & 4. & 5. & 6. & \\
\hline G & 48,03 & - & 48,48 & 48,27 & - & 48,15 & 48,35 \\
\hline $\mathrm{H}$ & 7,07 & $\div$ & 7,13 & 7,08 & - & $7,20 \quad-$ & 6,97 \\
\hline $\mathrm{N}$ & - & 15,85 & - & -1 & 15,08 & - $\quad 15,31$ & 15,38 \\
\hline
\end{tabular}

b) Antipepton $\alpha$ aus Eisenniederschlag II.

Darstellung B:

Das Präparat wurde in 2 Teilen gefällt.

I. Fällung. Das Präparat war völlig aschefrei.

1. 0,1942 g S. g. $\left\{\begin{array}{l}0,3284 \mathrm{~g} \mathrm{CO}_{2}=46,13 \% \mathrm{C} \\ 0,1088 \mathrm{~g} \mathrm{H}_{2} \mathrm{O}=6,39 \% \mathrm{H}\end{array}\right.$

2. $0,1779 \mathrm{~g} \mathrm{~S}$. g. $25 \mathrm{ccm} \mathrm{N}$ bei $20^{\circ}$ und $755,5 \mathrm{~mm}$ Bar.

$\mathrm{N}=16,60 \%$. 
3. 0,1752 g S. g. $24,1 \mathrm{ccm} \mathrm{N}$ bei $21^{\circ}$ und $763 \mathrm{~mm}$ Bar.

$$
\mathrm{N}=16,00 \% \text {. }
$$

II. Fällung.

Das Präparat war gleichfalls aschefrei.

4. 0,1397 g S. g. $\left\{\begin{array}{l}0,2362 \text { g CO } \\ 0,0853 \mathrm{~g} \mathrm{H}_{8} \mathrm{O}=66,11 \% \mathrm{C} \\ 6,84 \% \mathrm{H}\end{array}\right.$

5. 0,1377 g S. g. $19,3 \mathrm{ccm} \mathrm{N}$ bei $14^{\circ}$ und $748 \mathrm{~mm}$ Bar.

$$
\mathrm{N}=16,04 \% \text {. }
$$

Darstellung G (Methylalkoholmethode).

6. $0,2355 \mathrm{~g}$ S. g. $\left\{\begin{array}{l}0,3978 \mathrm{~g} \mathrm{CO}_{2}=46,22 \% \mathrm{C} \\ 0,1470 \mathrm{~g} \mathrm{H}_{2} \mathrm{O}=6,99 \% \mathrm{H}\end{array}\right.$

7. 0,2276 g S. g. $32,0 \mathrm{ccm} \mathrm{N}$ bei $14^{\circ}$ und $748 \mathrm{~mm}$ Bar.

\begin{tabular}{|c|c|c|c|c|c|c|c|c|}
\hline & \multicolumn{7}{|c|}{ Gefundene Prozente } & \multirow{2}{*}{$\begin{array}{c}\text { Berechnete } \\
\begin{array}{c}\text { Prozente } \\
\text { für }\end{array} \\
\end{array}$} \\
\hline & \multicolumn{3}{|c|}{$\begin{array}{l}\text { Präparat B. } \\
\text { I. Fäll. }\end{array}$} & \multicolumn{2}{|c|}{$\begin{array}{l}\text { Präparat B. } \\
\text { II. Fäll. }\end{array}$} & \multicolumn{2}{|c|}{ Präparat C. } & \\
\hline No. & 1. & 2. & 3. & 4. & 5. & 6. & 7. & $\mathrm{C}_{10} \mathrm{~N}_{8} \mathrm{H}_{17} \mathrm{O}_{5}$ \\
\hline C & 46,13 & - & - & 46,11 & - & 46,22 & - & 46,33 \\
\hline $\mathrm{H}$ & 6,39 & - & - & 6,84 & - & 6,99 & - & 6,56 \\
\hline $\mathrm{N}$ & - & 16,60 & 16,00 & - & 16,04 & - & 16,46 & 16,21 \\
\hline
\end{tabular}

$N^{\dagger}=16,46 \%$. Aschegehalt $0,4 \%$.

Zur Darstellung der Salze der Antipeptone wurden die bei der Polarisation benutzten Lösungen verwendet. Das Zinksalz wurde durch Aufkochen mit reinem Zinkoxyd, das Barytsalz durch Zusatz von überschüssigem Barytwasser in der Kälte, Einleiten von Kohlensäure und Aufkochen gewonnen.

Der Peptongehalt der Polarisationslösungen war zu gering, um die Salze aus den Lösungen mit Alkohol zu fällen. Sie wurden vielmehr direkt bei niederer Temperatur eingedampft, bei $70^{\circ}$ bis zur Konstanz getrocknet und dann verascht.

1. Baryumsalz aus Antipepton $\beta$ : 0,2629 g S. g. $0,0902 \mathrm{~g} \mathrm{BaSO}_{4}=0,0529 \mathrm{~g} \mathrm{Ba}$

Gefunden: Berechnet für $\left(\mathrm{C}_{11} \mathrm{H}_{18} \mathrm{~N}_{3} \mathrm{O}_{6}\right)_{\mathbf{8}} \mathrm{Ba}$ $\% \mathrm{Ba}=20,16 \quad 20,16$.

2. Zinksalz aus Antipepton $\alpha$ : 0,2317 g S. g. 0,0312 g ZnO $=0,0251$ g Zn

Gefunden:

$\% \mathrm{Zn}=10,83$
Berechnet für $\left(\mathrm{C}_{10} \mathrm{~N}_{8} \mathrm{O}_{5} \mathrm{H}_{16}\right)_{2} \mathrm{Zn}$

11,25 . 
3. Baryumsalz aus Antipepton $\alpha$ nach zweimaligem Umfällen des Peptons:

$$
\begin{aligned}
& \text { 0,3815 g S. g. } 0,1380 \mathrm{~g} \mathrm{BaSO}_{4} \\
& \begin{array}{l}
\text { Gefunden: } \\
\text { Berechnet für }\left(\mathrm{C}_{10} \mathrm{~N}_{3} \mathrm{O}_{5} \mathrm{H}_{16}\right)_{2} \mathrm{Ba}
\end{array} \\
& 21,03 .
\end{aligned}
$$
fällen:

4. Baryumsalz aus Antipepton $\alpha$ nach 3 maligem Um-

$$
\begin{aligned}
& \text { 0,2786 g S. g. } 0,0986 \text { g BaSO }_{4} \\
& \begin{array}{l}
\text { Gefunden: } \\
\% \mathrm{Ba}=20,82
\end{array} \quad \text { Berechnet für }\left(\mathrm{C}_{10} \mathrm{~N}_{8} \mathrm{O}_{6} \mathrm{H}_{16}\right)_{2} \text { Ba } \\
& 21,03 .
\end{aligned}
$$

Die Reaktionen der Antipeptone waren folgende:

1. Die Millonsche Reaktion: negativ.

2. Molischsche Reaktion: negativ, in einem Falle in konzentrierter Lösung schwach positiv.

3. Biuret-Reaktion: stark positiv.

4. Ferrocyankalium und Essigsäure: geben keine Trübung.

5. Bleiessig: fällt nicht in verdünnter Lösung (in einem Fall auch in konz. Lösung nicht).

6. Pikrinsäure: gibt eine geringe Trübung, die beim Erhitzen verschwindet und beim Erkalten wieder erscheint (in einem Falle auch in konz. Lösung keine Trübung).

7. Gerbsäure: gibt in nicht zu verdünnter Lösung eine Fällung, die in Essigsäure löslich ist.

8. Metaphosphorsäure: gibt keine Trübung.

9. Sublimat: gibt ganz schwache Trübung.

10. Phosphorwolframsäuse: gibt in konzentrierter Lösung eine Fällung, in verdünnter nicht.

\section{Xanthoprotein-Reaktion: positiv.}

Nach diesen Übereinstimmungen in Zusammensetzung, Salzbildung und Reaktionen kann kein Zweifel mehr vorliegen, daß die von mir aus Fibrin dargestellten Antipeptone rein waren.

\section{Nachweis der Konstanz des spez. Drehvermögens der Antipeptone.}

Die beiden Antipeptone drehen die Ebene des polarisierten Lichtes nach links.

Für das Umfällen der Substanz hat es sich im Laufe der Untersuchungen im Laboratorium als am zweckmäßigsten er- 
wiesen, 2 Teile des Peptons in 3 Teilen Wasser zu lösen und dann portionsweise 2 Teile absoluten Alkohol zuzugeben. Verrührt man diese Lösung in Alkohol, so fällt das ursprünglich weißflockige Pepton in kleinen, mitunter leicht gefärbten Nädelchen (keine Krystalle), die gut absitzen und sich sehr bequem absaugen und waschen lassen. - Ein Zusatz von Essigsäure zu der Lösung, der bei der Fällung des ersten, im Vacuum gewonnenen Sirups nötig ist, um das noch zum Teil als Ammonsalz vorliegende Antipepton zu zersetzen, schien bei der Umfällung an sich nicht erforderlich $z u$ sein, wenn man das Pepton möglichst vor Anziehung von Ammoniak aus der Luft geschützt hatte.

Es wurde die bemerkenswerte Tatsache beobachtet, dass wenn das Pepton aus rein wässeriger Lösung umgefällt wurde, das Drehvermögen anstieg; wurde hingegen eine Spur Essigsäure zugesetzt, so sank das Drehvermögen auf den konstanten Wert zurück.

Der Volumenprozentgehalt der polarisierten Lösungen wurde vorwiegend durch Trocknen abgewogener Substanzmengen bei $70^{\circ}$ bis zur Gewichtskonstanz, Lösen und Auffüllen auf ein gemessenes Volumen bestimmt. In einigen Fällen wurde die Substanz bloß zur Verjagung des Alkohols einige Tage im Trockenschrank gehalten, gelöst, polarisiert und durch Eindampfen eines gemessenen Volumens der Prozentgehalt bestimmt.

\section{Spez. Drehvermögen von Antipepton $\beta$.}

1. $1,0965 \mathrm{~g}$ Antipepton $\beta$ aus Darstellung A wurden im $50 \mathrm{ccm}-$ Kölbchen gelöst. Prozentgehalt mithin 2,193. Drehung im $1 \mathrm{dm}-\mathrm{Rohr}$ $0,71^{\circ}$ nach links. $t=18^{\circ}$.

Hieraus ergibt sich:

$$
[\alpha]_{\mathrm{D}}^{18}=-32,38^{\circ}
$$

2. $0,3838 \mathrm{~g}$ Antipepton $\beta$ aus Darstellung $\mathrm{B}$ wurden im $25 \mathrm{ccm}$ Kölbchen gelöst. Prozentgehalt 1,5352. Die Ablenkung im $2 \mathrm{dm}-\mathrm{Rohr}$ betrug $1,00^{\circ}$ n. $1 . \quad t=20^{\circ}$

$$
[\alpha]_{\mathrm{D}}^{20}=-32,57^{\circ}
$$

$8 \mathrm{~g}$ dieses Präparates wurden 4 mal umgefällt und zwar die ersten 3 Male ohne Zusatz von Essigsäure zu der wässerigen Lösung. 
3. Das Präparat wurde nach der 2. Umfällung polarisiert. Die $2,623 \%$ ige Lösung drehte im $2 \mathrm{dm}-\operatorname{Rohr} 1,45^{\circ}$ n. $1 . t=22^{\circ}$

$$
[\alpha]_{\mathrm{D}}^{22}=-35,84^{\circ} \text {. }
$$

4. Polarisation der 3. Umfällung: Die 1,357\% ige Lösung drehte im $2 \mathrm{dm}-\operatorname{Rohr} 1,11^{\circ}$ n. $1 . t=20^{\circ}$

$$
[\alpha]_{D}^{22}=-40,9^{\circ} \text {. }
$$

Es stieg also die spez. Drehung des Präparates durch 3 maliges Umfällen von $-32,38^{\circ}$ auf $-40,9^{\circ}$. Die empirische Zusammensetzung hatte sich dabei nicht geändert, wie die Analyse auf S. 272 ergibt.

Jetzt wurde das 3 mal umgefällte Präparat aus wässeriger Lösung unter Zusatz einer Spur Essigsäure umgefällt. Die Polarisation dieser 4. Umfällung ergab:

5. $0,3140 \mathrm{~g}$ wurden im $25 \mathrm{ccm}$-Kölbchen gelöst. Prozentgehalt 1,256. Ablenkung im $2 \mathrm{dm}$-Rohr $0,81 \mathrm{n}$. $1 . \mathrm{t}=20^{\circ}$

$$
\left[\alpha \left[_{D}^{20}=-32,25^{\circ}\right.\right. \text {. }
$$

Somit war durch die Einwirkung der Essigsäure der Wert für die spez. Drehung mit einem Schlage auf den konstanten Wert zurückgegangen.

Die Vermutung lag nahe, daß in den Fällen 3 und 4 eine Aufnahme von Ammoniak die Drehung der Polarisationsebene erhöht habe, da ja die Peptone leicht beim Stehen an der Luft Ammoniak binden.

6. Zum Versuch wurde die Lösung der Umfällung II, die verschlossen bei Zusatz eines Tropfens Chloroform aufbewahrt worden war, mit einem Tropfen konzentriertem Ammoniak versetzt, sodaß die Lösung alkalisch reagierte, und polarisiert:

Prozentgehalt der Lösung 2,023. Ablenkung im 2 dm-Rohr 1,440 nach links. $t=20^{\circ}$

$$
[\alpha]_{D}^{20}=-35,59^{\circ} \text {. }
$$

Jetzt wurden von der Umfällung IV (mit Essigsäure umgefällt) $15 \mathrm{ccm}$ der Lösung mit $5 \mathrm{ccm}$ konzentriertem Ammoniak versetzt und polarisiert:

7. Prozentgehalt 0,942. Die Ablenkung betrug im $2 \mathrm{dm}-$ Rohr 0,54 n. $1 . t=20^{\circ}$

$$
[\alpha]_{D}^{20}=-28,66^{0} .
$$

Die Drehung war also in ammoniakalischer Lösung keineswegs erhöht, sondern sogar erniedrigt worden.

Zum Beweis, daß das mit Essigsäure umgefällte Prä̀parat 
durch sorgsames Auswaschen absolut von Essigsäure befreit war, wurde die polarisierte Lösung mit Schwefelsäure im Überschuß versetzt und destilliert. Das Destillat gab auf Lackmus keine Säurereaktion. Es wurde mit 2 Tropfen Sodalösung zur Trokne gedampft und die Kakodylprobe angestellt. Sie fiel absolut negativ aus. - Somit war bewiesen, daß eine Verunreinigung mit Essigsäure nicht die Änderung der spez. Drehung hervorgerufen hat.

Beim Umfällen der Antipeptone aus wässeriger Lösung scheint demnach eine intramolekulare Umwandlung des Antipeptons $\beta$ einzutreten, die durch die Einwirkung einer Spur Essigsäure wieder aufgehoben wird.

Das spez. Drehvermögén von Antipepton $\beta$ a us Fibrin beträgt.

$$
[\alpha]_{D}^{20}=-32,40 .
$$

Spez. Drehvermögen von Antipepton $\alpha$.

Die Untersuchungen des spez. Drehvermögens von Antipepton $\alpha$ wurden an den Präparaten aus Darstellung B und Darstellung C. (mit der Methylalkoholmethode aus Wittepepton) angestellt.

Alle Umfällnngen wurden unter Zusatz einer Spur Essigsäure vorgenommen. Die Drehung des ersteren Präparates wurde erst nach der zweiten Umfällung konstant.

$5 \mathrm{~g}$ Antipepton $\alpha$ aus Darstellung B wurden $3 \mathrm{mal}$ aus wässeriger Lösung mit Essigsäurezusatz umgefällt und die einzelnen Fällungen polarisiert.

8. I. Umfällung: Die 1,4410\% ige Lösung drehte im $2 \mathrm{dm}$-Rohr $0,570 \mathrm{n}, \mathrm{l} . \mathrm{t}=20^{\prime}$

$$
[\alpha]_{D}^{20}=-19,77^{\circ} .
$$

9. II. Umfällung: $0,3713 \mathrm{~g}$ wurden im $25 \mathrm{ccm}-$ Kölbchen gelöst. Prozentgehalt 1,4852, Ablenkung im 2 dm-Rohr $0,73^{\circ}$ n. $1 . t=20^{\circ}$

$$
[\alpha]_{\mathrm{D}}^{20}=-24,57^{\circ} \text {. }
$$

10. III. Umfällung: $0,5107 \mathrm{~g}$ gelöst im $25 \mathrm{ccm}-K o ̈ l b c h e n$. Prozentgehalt 2,0428. Ablenkung im $2 \mathrm{dm}-\mathrm{Rohr} 1,00 \mathrm{n}$. $1 . t=20^{\circ}$

$$
[\alpha]_{D}^{20}=-24,47^{\circ} \text {. }
$$

11. Vom Antipepton $C_{i} \alpha$, aus Wittepepton gewonnen, wurden $0,48,76 \mathrm{~g}$ im $25 \mathrm{ccm}-K o ̈ l b c h e n$ gelöst. Prozentgehalt 1,9504. Die $\mathrm{Ab}-$ lenkung im $2 \mathrm{dm}$-Rohr betrug 0,97 n. $1 . t=20^{\circ}$

$$
[\alpha]_{D}^{20}=-24,87^{\circ} \text {. }
$$


Das spez. Drehvermögen von Antipepton $\alpha$ ist som it

$$
[\alpha]_{\mathrm{D}}^{20}=-24,5^{\circ} \text {. }
$$

$\mathrm{Daß}$ eine Konzentrationsverschiedenheit der Lösungen zwischen $3 / 4$ und $2 \%$ ohne Einfluß auf das spez. Drehvermögen der Antipeptone ist, wurde in mehreren Fällen durch Polarisieren der Lösungen nach geeigneter Verdünnung bewiesen. Die gefundenen Werte blieben innerhalb der Beobachtungsfehler stets konstant.

12. Die Lösung 3 wurde auf das doppelte Volumen verdünnt. Prozentgehalt 1,0115. Ablenkung im $2 \mathrm{dm}-\operatorname{Rohr} 0,72^{\circ} \mathrm{n} .1 . \mathrm{t}=20^{\circ}$

$$
[\alpha]_{D}^{20}=-35,59^{\circ} \text {. }
$$

13. Die Lösung 10 wurde in halber Konzentration polarisiert. Prozentgehalt 0,7426. Ablenkung im $2 \mathrm{dm}-$ Rohr $0,37^{\circ} \mathrm{n} .1$.

$$
[\alpha]_{\mathrm{D}}^{20}=-24,91^{\circ} \text {. }
$$

14. Die Lösung 11 wurde bei einem Prozentgehalt von 0,9752 polarisiert. Ablenkung im $2 \mathrm{dm}-\operatorname{Rohr} 0,48^{\circ} \mathrm{n}$. $1 . \mathrm{t}=20^{\circ}$

$$
[\alpha]_{\mathrm{D}}^{20}=-24,61^{\circ} \text {. }
$$

\section{Spaltungen der Antipeptone.}

In der Einleitung habe ich bereits die Hauptzwecke der angestellten Spaltungsversuche angegeben. Vorangestellt sein möge nochmals, was Siegfried betont hat, daß es fraglich ist, ob bei der Spaltung durch siedende Säure bloß eine Trennung der typischen, am Aufbau des Antipeptonmoleküls beteiligten Gruppen eintritt; "denn viel näher, als die Vorstellung, daß das Eiweißmolekül etwa nur aus Anhydriden von Amidosäuren und Basen besteht, liegt die Annahme, daß bei der Spaltung der Proteinstoffe durch Mineralsäuren auch Kohlenstoffketten und Kohlenstoff-Stickstoff-Verkettungen gespalten werden, daß diese Spaltungen teils an dieser, teils an jener Stelle ansetzen, sodaß die entstehenden Spaltungsprodukte in Summa viel mehr C-Atome enthalten als das ursprüngliche Eiweißmolekül.»

24,5 g trockenes Antipepton $\alpha$ wurden mit der dreifachen Menge konz. Schwefelsäure und der 6 fachen Menge $\mathrm{W}^{\top}$ assers 12 Stunden am Rückflußkühler zum Sieden erhitzt. Die 
Lösung wurde aufs 4 fache Volumen verdünnt und filtriert, wobei ein Körper von der Art der Schmiedebergschen Melanoidinsäure nicht zurückblieb; die Lösung war bis auf ganz geringe Spuren klar.

Die Spaltungslösung wurde mit 50\% iger Phosphorwolframsäure gefällt.

a) Der Phosphorwolframsäureniederschlag.

Der abgesaugte und mit kalter 5\% iger Schwefelsäure gewaschene Niederschlag der Basen wurde in Wasser verrührt, auf $40^{\circ}$ erwärmt und mit Hilfe von Ammoniak in Lösung gebracht. Die Phosphorwolframsäure und Schwefelsäure wurden durch Fällen mit Baryt entfernt, das Filtrat durch Ammoncarbonat von dem geringen Überschuß des Baryts befreit und die Lösung auf dem Wasserbad eben zur Trockne gedampft. Der Rückstand wurde mit $100 \mathrm{ccm}$ Wasser aufgenommen, in der Schüttelflasche solange mit Silbernitratlösung versetzt, bis ein Tropfen mit Barytwasser Gelbfärbung gab, und hierauf mit gepulvertem Baryt gesättigt. Der entstandene, schwarze Niederschlag wurde sofort abgesaugt, mit gesättigtem Barytwasser gewaschen und auf Arginin und Histidin untersucht.

Er wurde in Wasser verrührt und mit Schwefelsäure und Salzsäure zur Entfernung des Baryts und Silbers versetzt; das Filtrat wurde wieder mit Phosphorwolframsäure gefällt. Der Niederschlag wurde abgesaugt und mit kalter 5\% oiger Schwefelsäure gewaschen. Dann wurde er wie oben in wässerigem Ammoniak gelöst, mit Baryt zersetzt und nach Entfernung des Überschusses mit Ammoncarbonat auf dem Wasserbad zur Trockne gedampft. Er wog 1,7 g. Der in wenig Wasser gelöste Rückstand wurde in konz. Salpetersäure bis eben zur saueren Reaktion auf Kongopapier und alsdann mit 2 g Silbernitrat versetzt. Es entstand eine schwache Trübung. Auf Zusatz der 10fachen Menge Alkohol schieden sich spärlich braune Flocken ab, von denen abfiltriert wurde und deren Untersuchung auf Histidin wegen ihrer geringen Menge unterbleiben mußte. Zum Filtrat wurde Äther gesetzt, worauf eine 
weiße Trübung entstand, die aber beim Stehen über Nacht sich in Schmieren am Boden niedersetzte. Trotz längeren Stehens in der Kälte und portionsweisen Zusatzes von Äther gelang es nicht, Krystalle zu erhalten. Deshalb wurde das Ganze nochmals eingedampft, in möglichst wenig Wasser und etwas wässerigem Silbernitrat aufgenommen, mit dem 10fachen Volumen Alkohol versetzt und Äther zugegeben, während die Lösung noch etwas warm war. Es entstand eine bräunliche Trübung, die beim Stehen gelb gefärbte Krystalle in kugeligen Aggregaten absetzte. Auf portionsweisen Zusatz von Äther setzten sich alsdann darüber weiße Nadeln ab. Diese wurden abgesaugt und mit Äther gewaschen. Nach einmaligem Umkrystallisieren wurden rein weiße Krystalle von der Zusammensetzung und dem Schmelzpunkt des saueren Arginin-Silbernitrats erhalten. Die Ausbeute betrug $0,8 \mathrm{~g}$.

Zur Analyse wurde die Substanz im Vacuum über Schwefelsäure getrocknet.

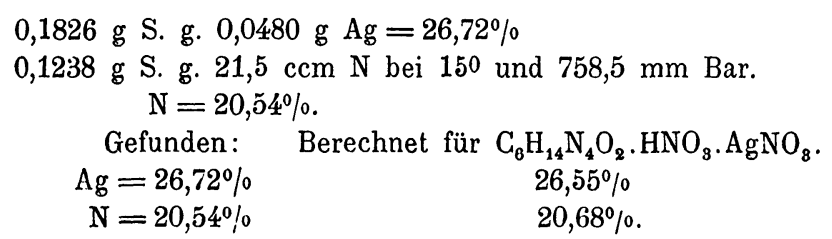

Die Schmelzpunktbestimmung ergab 176-176,50 (uncorr.), in Übereinstimmung mit den Beobachtungen von Gule witzs ch, $\left.{ }^{1}\right)$ der für 3 Präparate von sauerem Argininsilbernitrat (aus tierischem Eiweiß) $180-183^{\circ}, 180-181^{\circ}$ und $176-177^{\circ}$ fand.

Von einer Bestimmung des optischen Drehvermögens mußte leider abgesehen werden, da die mir nach einem Verluste von $0,2 \mathrm{~g}$ noch verbleibende Menge bei dem niedrigen Drehvermögen zur Bestimmung nicht ausreichte.

Das Filtrat vom Argininniederschlag wurde gleichfalls mit Schwefelsäure und Salzsäure von Baryt und Silber befreit und wiederum mit Phosphorwolframsäure gefällt. Der Niederschlag wurde in Wasser verrührt, bei $40^{\circ}$ in Ammoniak gelöst, mit Baryt zersetzt und der Überschuß von Baryt mit Ammon-

1) Gulewitzsch, Diese Zeitschr., Bd. XXVII, S. 200. 
carbonat entfernt. Das Filtrat wurde zur Trockne gedampft; der Rückstand wog 1,5 g. - Durch analoge Behandlung wie beim Arginin wurde ein Silbersalz hergestellt. Die alkoholische Lösung schied auf Zusatz von Äther Schmieren $a b$, die bei einem gewissen Verhältnis von Alkohol und Äther fest wurden, aber nicht krystallinisch waren. - Nach vergeblichen Versuchen, Krystalle von Lysinsilber zu erhalten, wurde zur Trockne gedampft, in Wasser gelöst, mit Salzsäure zersetzt und das Filtrat mit Phosphorwolframsäure gefällt. Der Niederschlag wurde wie oben zersetzt und eingedampft. Der Rückstand wog noch $0,6 \mathrm{~g}$. Er wurde in wenig Wasser gelöst, mit $2 \mathrm{~g}$ festem Platinchlorid versetzt und mit Alkohol gefällt.

Schon nach 10 Minuten hatten sich eine Menge gelber Nadeln abgesetzt, die nach mehrtägigem Stehen abgesaugt, gewaschen und über Schwefelsäure getrocknet wurden. Ihr Gewicht betrug $1,3 \mathrm{~g}$.

0,2016 g S. g. 0,0656 g Pt.

Gefunden: Berechnet für $\mathrm{C}_{8} \mathrm{~N}_{2} \mathrm{H}_{14} \mathrm{O}_{2} \cdot 2 \mathrm{HCl} \cdot \mathrm{PtCl}_{4}+\mathrm{C}_{2} \mathrm{H}_{5} \mathrm{OH}$ $\% \mathrm{Pt}=32,54$

32,37 .

Da die Entstehung von Lysin bereits von Siegfried endgültig bewiesen war, begnügte ich mich mit der Platinbestimmung zur Identifizierung des Lysinchloroplatinats.

b) Das Filtrat vom Phosphorwolframsäureniederschlag.

Das Filtrat vom Phosphorwolframsäureniederschlag wurde mit Baryt gefällt, abgesaugt und mit Ammoncarbonat vom überschüssigen Baryt befreit. Das Filtrat wurde zum Sirup eingedampft; nach einigem Stehen erfolgte Krystallausscheidung, die sich in Wasser bis auf geringe Mengen eines schwarzen Rückstands löste. Aus der Lösung wurde die Glutaminsäure mit ammoniakalischer Silberlösung abgeschieden. Der starke Niederschlag wurde mit Wasser bis zum Schwinden der Salpetersäurereaktion gewaschen, alsdann in Wasser suspendiert und mit Salzsäure im geringen Überschuß geschüttelt. Das Filtrat vom Chlorsilber wurde eingedampft und gab einen äußerst zähen, braungelben Sirup. Dieser wurde aus der Schale herausgekratzt, die Reste mit konz. Salzsäure und. 
wenig Wasser herausgelöst. Zu dem Ganzen wurde soviel Wasser gegeben, daß sich die festen Anteile eben lösten. Jetzt wurde unter guter Kühlung Salzsäuregas bis zur Sättigung eingeleitet; nach 2tägigem Stehen im Eis hatten sich rein weiße Krystalle abgeschieden, die über Asbest abgesaugt und mit konz. Salzsäure gut gewachsen wurden. Ihr Gewicht betrug im Vacuum getrocknet 2,7 g. Aus der Mutterlauge schieden sich allmählich weitere, unreine Krystalle ab, deren Menge nach 10wöchentlichem Stehen im Eisschrank $1 \mathrm{~g}$ betrug. Die gefundene Ausbeute an Glutaminsäure aus Antipepton $\alpha$ berechnet sich hieraus zu 12\%.

Zur Analyse wurde die Substanz über Kalk getrocknet.

$$
\begin{aligned}
& 0,2200 \text { g S. g. } 0,1749 \mathrm{~g} \mathrm{AgCl} \\
& 0,3913 \text { g S. sättigten } 21,64 \mathrm{ccm} \quad{ }^{\mathrm{n}} /{ }_{10} \mathrm{H}_{2} \mathrm{SO}_{4} \\
& \quad=0,03029 \mathrm{~g} \mathrm{~N} \quad \mathrm{~N}=7,63 \% .
\end{aligned}
$$

Gefunden:

$\% \mathrm{Cl}=19,62$

$\% \mathrm{~N}=7,63$
Berechnet auf $\mathrm{C}_{5} \mathrm{H}_{9} \mathrm{NO}_{4} \cdot \mathrm{HCl}$

19,35

7,74 .

Zur Polarisation der salzsauren Glutaminsäure wurde eine annähernd $4^{0} /$ ige wässerige Lösung benutzt, deren spez. Drehvermögen nach den Angaben von Scheibler $\left.{ }^{1}\right)+20,4^{0}$ sein soll, woraus sich für die spez. Drehung von Glutaminsäure der Wert + 25,50 berechnet. - Meine Bestimmung des Drehvermögens von salzsaurer Glutaminsäure ergab einen höheren Wert, $+24,52^{\circ}$, aus dem sich ein Wert für Glutaminsäure berechnet $\left(+30,62^{\circ}\right)$, der gut zu den von E. Fischer gefundenen Wierten von $28-32^{\circ}$ paßt.

Andere Bestimmungen des spez. Drehvermögens von Glutaminsäurechlorhydrat habe ich in der Literatur nicht gefunden.

Die 3,913\% \% ige Lösung drehte im 2 dm-Rohr bei $20^{\circ}$ $1,92^{\circ}$ n. r.

$$
\alpha_{[D]}^{20}=+24,520 .
$$

Hieraus herechnet sich für Glutaminsäure in salzsaurer Lösung:

$$
\alpha_{[D]}^{20}=+30,620 .
$$

Ein Teil der salzsauren Glutaminsäure wurde in wenig

1) C. Scheibler, Untersuchungen über die Glutaminsäure. Berichte d. deutsch. chem. G. Bd. 17 S. 1728. 
heißem Wasser gelöst und tropfenweise mit Ammoniak bis zur eben alkalischen Reaktion versetzt. Die auskrystallisierte Glutaminsäure war nach 2maligem Umkrystallisieren chlorfrei.

Zum Vergleich mit den in neuster Zeit von E. Fischer und seinen Schülern angestellten Untersuchungen wurde eine Polarisation der Glutaminsäure unter denselben Versuchsbedingungen angestellt.

0,2238 g. S. wurden im $25 \mathrm{ccm}$-Kölbchen in $20 \%$ iger Salzsäure gelöst. Prozentgehalt 0,8952 .

Die Ablenkung im $2 \mathrm{dm}$-Rohr betrug $0,54^{\circ}$ n. r. $t=20^{\circ}$

$$
\alpha_{[D]}^{20}=+30,17^{\circ}
$$

Zum Vergleich seien die von $\mathrm{E}$. Fis $\mathrm{ch} \mathrm{er}^{1}$ ) gefundenen Werte daneben gestellt.

$$
\begin{array}{r}
\text { Glutaminsäure aus Horn }+31,91^{\circ} \\
\text { aus Gelatine }+30,85^{\circ} \\
\text { aus Casein }+28,21^{\circ} .
\end{array}
$$

Die Mutterlauge von der salzsauren Glutaminsäure wurde auf dem Wasserbad zum Sirup gedampft; Krystallausscheidung erfolgte nicht. Der Rückstand löste sich ganz in Alkohol und wurde mit alkoholischem Ammoniak versetzt; es entstand ein grauer Niederschlag, der abgesaugt, gewaschen und getrocknet wurde. Der Körper war weder ans Wasser, noch aus Alkohol krystallinisch zu erhalten. Wahrscheinlich enthielt er Reste der Glutaminsäure.

Aus dem Filtrat wurde mit alkoholischem Kupferacetat eine Fällung erhalten, die aus Wasser auszukrystallisieren versucht wurde. Nach wochenlangem Stehen setzten sich eine geringe Menge kleiner, blaugrüner Nädelchen ab. Die Mutterlauge schied beim Einengen stets bloß amorphe Flocken ab (0,3 g), die auch aus Alkohol nicht krystallisierten. Die Krystalle wurden analysiert, wenngleich bei ihrer geringen Menge der Bestimmung keine absolute Sicherheit zugesprochen werden kann.

$0,0534 \mathrm{~g}$ lufttrockenes Salz gaben bei $130^{\circ}$ getrocknet $0,0161 \mathrm{~g} \mathrm{H}_{2} \mathrm{O}$ ab und 0,0131 $\mathrm{g} \mathrm{Cu}$. Seite 475 .

1) E. Fischer und Dörpinghaus, Diese Zeitschr., Bd. XXXVI, 


$$
\begin{array}{cc}
\text { Gefunden: } & \text { Berechnet für } \mathrm{C}_{4} \mathrm{H}_{5} \mathrm{NO}_{4} \mathrm{Cu}+4^{1} / 2 \mathrm{H}_{2} \mathrm{O} \\
\% \mathrm{H}_{2} \mathrm{O}=30,15 & 29,45 \% \\
\% \mathrm{Cu}=24,50 & 23,02 \% .
\end{array}
$$

Der. unter den Umständen ziemlich genaue Wasserwert macht es wahrscheinlich, daß hier Asparaginsäure vorliegt, da das Kupfersalz der Asparaginsäure allein unter den hier in Betracht kommenden Amidosäuren mit so viel Molekülen Wasser krystallisiert.

Das Filtrat von der mit ammoriakalischer Silberlösung abgeschiedenen Glutaminsäure wurde mit Schwefelammon vom Silber befreit und das Filtrat eingedampft. Der Rückstand wurde einige Stunden am Rückflußkühler mit Alkohol ausgekocht. Der Auszug gab mit alkoholischem Silbernitrat nur eine geringe Fällung. Der größte Teil war ungelöst geblieben; er wurde in Wasser aufgenommen und heiß in siedenden Alkohol verrührt. Es. setzten sich fest am Boden haftende Schmieren ab, die im Verlauf von 14 Tagen anfingen, kugelige Krystalle anzusetzen. Die Krystalle waren jedoch nicht mechanisch von den Schmieren zu trennen. Da sie auch nach mehrfachem Umfällen nicht ohne beigemengte, unkrystallinische Teile zu erhalten waren, wurden Krystalle und Schmieren wieder in Wasser gelöst und in das Kupfersalz übergeführt. Dies geschah durch Kochen der Lösung mit überschüssigem, frisch gefälltem anhydrischen Kupferoxydhydrat.

Die Lösung war tiefblau und blieb beim Konzentrieren klar. Als sich aus der wässerigen Lösung Krystalle nach 2 Tagen nicht abgeschieden hatten, wurde mit Alkohol versetzt. Es erfolgte in wenigen Minuten eine reichliche $\mathrm{Ab}$ scheidung hellblauer Krystalle. Nach 24stündigem Stehen wurde abgesaugt, mit Alkohol gewaschen und im Vacuum über Schwefelsäure getrocknet. Die Krystalle zeichneten sich durch Leichtigkeit aus.

Die Analyse ergab Werte, die auf Serinkupfer stimmen; der Kupfergehalt wurde indessen zu hoch gefunden.

Die Substanz wurde zunächst über Schwefelsäure zur Konstanz und dann im Luftbad bei $100^{\circ}$ getrocknet, wobei keine weitere Gewichtsabnahme erfolgte. 
$0,1309 \mathrm{~g} \mathrm{~S} . \mathrm{g} \cdot\left\{\begin{array}{l}0,1288 \mathrm{~g} \mathrm{CO}_{2} \\ 0,0416 \mathrm{~g} \mathrm{CuO}\end{array}\right.$

0,1784 g S. g. $16,5 \mathrm{ccm} \mathrm{N}$ bei $15^{\circ}$ und $761 \mathrm{~mm}$ Bar.

$\begin{array}{lcc} & \text { Gefunden: } & \text { Berechnet auf }\left(\mathrm{C}_{3} \mathrm{H}_{6} \mathrm{NO}_{3}\right)_{2} \mathrm{Cu}: \\ \% \mathrm{C}=26,82 & 26,51 \\ \% \mathrm{H}=4,49 & 4,32 \\ \% \mathrm{~N}=10,97 & 10,31 \\ \% \mathrm{Cu}=25,39 & 23,42\end{array}$

Leider war aus Mangel an Material eine weitere Untersuchung des Körpers nicht möglich.

Herr Prof. Siegfried ermächtigt mich, mitzuteilen, daß er auch aus Antipepton $\beta$ durch Spaltung mit Salzsäure Arginin erhalten hat.

Das Argininsilbernitrat lieferte folgende Werte bei der Analyse:

I. 0,2316 g S. g. 0,0810 g $_{2} 0$ u. $0,1523 \mathrm{~g} \mathrm{CO}_{2}$ u. $0,0620 \mathrm{~g} \mathrm{Ag.}$

II. $0,1861 \mathrm{~g} \mathrm{~S}$. g. $33,3 \mathrm{ccm}$ tr. $\mathrm{N}$ bei $755 \mathrm{~mm}$ u. $21^{\circ}$.

$\begin{array}{lc}\text { Gefunden: } & \text { Berechnet } \\ \% \mathrm{C}=17,94 & 17,68 \\ \% \mathrm{H}=3,88 & 3,71 \\ \% \mathrm{~N}=20,77 . & 20,68 \\ \% \mathrm{Ag}=26,77 & 26,55\end{array}$

Schließlich wurden noch quantitative Spaltungen angeführt, die mit neuem Material fortgesetzt werden sollen. Jetzt sei nur mitgeteilt, daß Antipepton $\beta 16,1 \%$, Antipepton $\alpha$ $21,9 \%$ seines Stickstoffes bei der Zersetzung mit Schwefelsäure (1 Teil konzentrierte Schwefelsäure, 2 Teile Wasser) als $\mathrm{NH}_{3}$ abspaltete und ferner, daß bei beiden Antipeptonen der Basenstickstoff weniger als $25 \%$ des Gesamtstickstoffs betrug.

Es sei mir vergönnt, meinem hochverehrten Lehrer, Herrn Prof. M. Siegfried, auch an dieser Stelle für die Anregung zu der Arbeit, sowie für die mir stets liebenswürdigst gewährte Belehrung und Unterstützung bei derselben meinen herzlichsten Dank auszusprechen. 